\title{
Targeting the translational machinery in gastrointestinal stromal tumors (GIST) - a new therapeutic vulnerability
}

Donna M. Lee, ${ }^{1, \star}$ Angela Sun,,${ }^{1, \star}$ Sneha S. Patil, ${ }^{1}$ Lijun Liu, ${ }^{1}$ Aparna Rao,,${ }^{1,2}$ Parker Trent, ${ }^{1}$

Areej A. Ali, ${ }^{1}$ Catherine Liu, ${ }^{1}$ Jessica L. Rausch, ${ }^{1}$ Laura Presutti, ${ }^{1}$ Nduka Amankulor,${ }^{1,2, \dagger}$ Masahiro Shuda, ${ }^{3,4}$ Anette Duensing ${ }^{1,5}$

\author{
${ }^{1}$ Cancer Therapeutics Program \\ UPMC Hillman Cancer Center \\ Pittsburgh, PA, USA \\ ${ }^{2}$ Department of Neurological Surgery \\ University of Pittsburgh School of Medicine \\ Pittsburgh, PA, USA \\ ${ }^{3}$ Cancer Virology Program \\ UPMC Hillman Cancer Center \\ Pittsburgh, PA, USA \\ ${ }^{4}$ Department of Microbiology \& Molecular Genetics \\ University of Pittsburgh School of Medicine \\ Pittsburgh, PA, USA \\ ${ }^{5}$ Department of Pathology \\ University of Pittsburgh School of Medicine \\ Pittsburgh, PA, USA
}

\footnotetext{
${ }^{*}$ Co-first authors

† Current address: Department of Neurological Surgery, The University of Pennsylvania, 3 Silverstein Pavilion.
}

Running title: Protein synthesis inhibition in GIST

Key words: gastrointestinal stromal tumor (GIST), imatinib mesylate, homoharringtonine, protein synthesis inhibition

Corresponding author: Anette Duensing, UPMC Hillman Cancer Center, Research Pavilion, Suite G.17, 5117 Centre Avenue, Pittsburgh, PA 15213, USA. Phone: +1-412-623-5870; Fax: +1-412623-1010; E-mail: aduensin@pitt.edu

The authors declare that they have no competing financial interests. 


\section{ABSTRACT}

Although KIT-mutant GISTs can be effectively treated with tyrosine kinase inhibitors (TKIs), many patients develop resistance to imatinib mesylate (IM) as well as the FDA-approved later-line agents sunitinib, regorafenib and ripretinib. Resistance mechanisms mainly involve secondary mutations in the KIT receptor tyrosine kinase gene indicating continued dependency on the KIT signaling pathway. The fact that the type of secondary mutation confers either sensitivity or resistance towards TKIs and the notion that secondary mutations exhibit intra- and intertumoral heterogeneity complicates the optimal choice of treatment in the imatinib-resistant setting. Therefore, new strategies that target KIT independently of its underlying mutations are urgently needed. Homoharringtonine (HHT) is a first-in-class inhibitor of protein biosynthesis and is FDA-approved for chronic myeloid leukemia $(\mathrm{CML})$ that is resistant to at least two TKIs. HHT has also shown activity in KIT-mutant mastocytosis models, which are intrinsically resistant to imatinib and most other TKIs. We hypothesized that HHT could be effective in GIST through downregulation of KIT expression and subsequent decrease of KIT activation and downstream signaling. Testing several GIST cell line models, HHT led to a significant reduction in nascent protein synthesis and was highly effective in the nanomolar range in IM-sensitive and IM-resistant GIST cell lines. HHT treatment resulted in a rapid and complete abolishment of KIT expression and activation, while KIT mRNA levels were minimally affected. The response to HHT involved induction of apoptosis as well as cell cycle arrest. The antitumor activity of HHT was confirmed in a GIST xenograft model. Taken together, inhibition of protein biosynthesis is a promising strategy to overcome TKI resistance in GIST. 


\section{INTRODUCTION}

The majority of gastrointestinal stromal tumors (GIST) arise from oncogenic mutations in the KIT receptor tyrosine kinase gene ${ }^{1-3}$. Hence, most inoperable and/or metastatic GISTs can be successfully treated with imatinib mesylate $(\mathrm{IM}$; Gleevec®), a small molecule tyrosine kinase inhibitor (TKI) that targets KIT and leads to disease stabilization in more than $85 \%$ of the patients ${ }^{4,5}$. However, complete remissions are rare, and approximately $50 \%$ of the patients experience disease progression within the first two years of therapy ${ }^{6}$. Drug resistance is mostly due to secondary KIT mutations that prevent imatinib from binding ${ }^{7,8}$. Unfortunately, second- and thirdline treatments, the TKIs sunitinib (Sutent $\left.{ }^{\circledR}\right)$ and regorafenib (Stivarga $\left.{ }^{\circledR}\right)$, only offer limited additional benefit ${ }^{9-11}$. A fourth-line medication (ripretinib, Qinlock®) was recently approved, but development of resistance is still a problem ${ }^{12}$. Another small molecule inhibitor, avapritinib (Ayvakit@), was approved only for a subset of GIST that carry a certain PDGFRA (platelet-derived growth factor receptor alpha) mutation (PDGFRA D842V) ${ }^{13,14}$. Hence, new therapeutic approaches for GIST patients are still needed, in particular when TKI resistance has occurred.

GIST cells are highly dependent on KIT signaling for their survival and proliferation. This is exemplified by the fact that GIST cell apoptosis is not only induced by TKI-mediated inhibition ${ }^{4,15}$, but also by loss of KIT expression via other mechanisms, such as siRNA-mediated knockdown, treatment with HSP90 inhibitors or inhibition of KIT gene transcription ${ }^{16-19}$. In addition, resistance mechanisms mainly involve secondary KIT mutations that prevent drug binding, further pointing towards the continued dependency on an activated KIT signaling pathway 7,8. Because secondary mutations exhibit heterogenous sensitivity to later-line TKIs and also display intra- and intertumoral heterogeneity, treatment responses are likely to be uneven depending on the clonal composition of the tumor ${ }^{20}$. Therapeutic strategies that target KIT independently of its underlying mutation could potentially overcome these obstacles ${ }^{21}$. In this scenario, targeting KIT protein expression by inhibiting mRNA translation represents a promising 
therapeutic strategy ${ }^{22}$.

Homoharringtonine (HHT) is a cytotoxic alkaloid that is derived from the Japanese Plum Yew (cephalotaxus harringtonia) ${ }^{23-25}$. It was originally used in Chinese traditional medicine and is now produced as semisynthetic, purified compound (omacetaxine mepesuccinate/Synriboß; Teva Pharmaceuticals) ${ }^{22,26}$. HHT acts as a global protein translation inhibitor by interfering with the elongation step of protein biosynthesis. It competitively binds the peptidyl transferase A-site cleft of ribosomes, thereby preventing the incoming amino acid side chains of aminoacyl-tRNAs from attaching ${ }^{24,27}$. HHT/omacetaxine is FDA-approved for the treatment of patients with chronic myeloid leukemia $(\mathrm{CML})$ that are resistant and/or intolerant to at least two TKIs ${ }^{26,28}$. Further clinical and preclinical studies have shown HHT's activity in acute myeloid leukemia, VHL-mutant clear cell renal cell carcinoma as well as rhabdoid tumors ${ }^{25,29,30}$. Of note, HHT has also shown preclinical activity in KIT D816V-mutant mastocytosis models, a disease that is intrinsically resistant to imatinib and most other TKIs targeting $\mathrm{KIT}^{31}$.

Our study investigates whether HHT can target KIT in GIST cells by inhibiting protein translation. We hypothesized that treatment with HHT reduces KIT protein levels, thereby decreasing KIT activation as well as its downstream signaling cascades ultimately leading to GIST cell apoptosis in an imatinib-sensitive as well as imatinib-resistant setting.

\section{MATERIALS AND METHODS}

\section{Cell culture, inhibitor treatments}

The imatinib-sensitive human GIST cell lines GIST882 (homozygous mutation in KIT exon 13 pK642E; kindly provided by Jonathan A. Fletcher, Brigham and Women's Hospital, Boston, MA, USA) and GIST-T1 (heterozygous KIT exon 11 deletion pV560_Y578del; kindly provided by Takahiro Taniguchi, Kochi Medical School, Kochi, Japan) were derived from untreated metastatic 
GISTs ${ }^{32,33}$. Imatinib-resistant GIST cell lines GIST430 (heterozygous primary KIT exon 11 deletion [V560_L576del], secondary KIT exon 13 point mutation [V654A]) and GIST48 (homozygous primary KIT exon 11 point mutation [V560D], secondary KIT exon 17 point mutation [D820A]; both kindly provided by Jonathan A. Fletcher, Brigham and Women's Hospital, Boston, MA, USA) were derived from human GISTs that had developed clinical resistance to imatinib therapy ${ }^{18,19}$. All GIST cells were propagated as previously described ${ }^{15,18,19}$.

Homoharringtonine (HHT; Santa Cruz) treatments were performed at the indicated concentrations (in DMSO) compared to $0.1 \%$ DMSO for up to $72 \mathrm{~h}$. Treatment with imatinib mesylate (1 $\mu \mathrm{M}$ in DMSO; LC Laboratories) or sunitinib (1 $\mu \mathrm{M}$ in DMSO; LC Laboratories) served as control. Cycloheximide $(\mathrm{CHX})$ treatment was performed at $30 \mu \mathrm{g} / \mathrm{ml}$ in $\mathrm{dH}_{2} \mathrm{O}$ unless otherwise noted.

\section{Immunological and cell staining methods}

Protein lysates of cells growing as monolayer were prepared as described previously ${ }^{15}$. In brief, cells were scraped into lysis buffer (1\% NP-40, $50 \mathrm{mM}$ Tris-HCl pH 8.0, $100 \mathrm{mM}$ sodium fluoride, $30 \mathrm{mM}$ sodium pyrophosphate, $2 \mathrm{mM}$ sodium molybdate, $5 \mathrm{mM}$ EDTA, $2 \mathrm{mM}$ sodium orthovanadate) with protease inhibitors $(10 \mu \mathrm{g} / \mathrm{ml}$ aprotinin, $10 \mu \mathrm{g} / \mathrm{ml}$ leupeptin, $1 \mu \mathrm{M}$ phenylmethylsulfonyl fluoride). The suspension was incubated with shaking $\left(1 \mathrm{~h}, 4^{\circ} \mathrm{C}\right)$ and cleared by centrifugation (13,000 rpm, $\left.30 \mathrm{~min}, 4^{\circ} \mathrm{C}\right)$. The Bradford assay (Biorad) was used to determine protein concentrations, and $30 \mu \mathrm{g}$ of protein were loaded on $4-12 \%$ Bis-Tris gels (Invitrogen).

Primary antibodies used for immunoblotting were p4E-BP1 T37/46, 4E-BP1 (both Cell Signaling), actin (Santa Cruz), cleaved caspase 3 (Cell Signaling), cyclin A (Novocastra), pKIT Y719 (Cell Signaling), KIT (DakoCytomation/Agilent), p27 Kip1 (BD Biosciences Pharmingen), PARP (Invitrogen), pS6K T389, S6K (both Abcam).

Immunohistochemistry was performed on sections $(4 \mu \mathrm{m})$ of formalin-fixed, paraffinembedded (FFPE) mouse tumors from in vivo experiments. Slides were deparaffinized, 
underwent antigen retrieval and were incubated with primary antibodies (KIT, DakoCytomation/Agilent; Ki-67, GeneTex; cleaved caspase 3, Cell Signaling). The signal was detected using biotin-conjugated secondary antibodies (Abcam), POD-streptavidin (Roche), and 3'diaminobenzidine-tetrahydrochloride (DAB) chromogen (Abcam). For Ki-67 and cleaved caspase 3 stains, the number of positive cells was counted in 10 high power fields (HFP) per tumor at 400 -fold magnification, with an average of 245 cells counted per HPF.

\section{Measurement of protein synthesis}

To analyze protein synthesis activity, cellular incorporation of L-homopropargylglycine (HPG), a methionine analogue with alkyne moiety was detected by Click-iT reaction with azidemodified Alexa $488{ }^{34}$. Cells treated with $\mathrm{HHT}$, imatinib or sunitinib for $1 \mathrm{~h}$ or $8 \mathrm{~h}$ were labelled with $25 \mu \mathrm{M}$ HPG (Invitrogen, C10102) for the last 30 min of the drug treatment in L-methioninedepleted medium containing 5\% dialyzed fetal calf serum (FCS; Gibco). Harvested cells were washed with PBS, fixed in $10 \%$ buffered formalin (4\% formaldehyde) for 5 min at room temperature and permeabilized for $30 \mathrm{~min}$ in PBS containing $0.1 \%$ saponine (Sigma) and $1 \%$ FBS (HyClone). The Click-iT reaction was performed for $30 \mathrm{~min}$ at room temperature by suspending the cells in Click-iT cell reaction buffer containing $1 \mathrm{mM}$ azide-modified Alexa 488 and $2 \mathrm{mM}$ CuSO4 (Invitrogen). Cells were washed twice in PBS with 1\% FCS, and the cell suspension was subjected to flowcytometry analysis. The protein synthesis inhibitor cycloheximide $(100 \mu \mathrm{g} / \mathrm{ml})$ was used as a positive control in this assay. Cells without Click-iT reaction were used as a background control.

\section{In vitro apoptosis and proliferation assays}

Apoptosis and cell viability studies were performed using the Caspase-Glo® and CellTiterGlo® luminescence-based assays (Promega) as described previously ${ }^{19}$. Cells were cultured in 96-well flat-bottomed plates and incubated with HHT (or DMSO-only solvent control) for 48h 
(Caspase-Glo®) or 72h (CellTiter-Glo®). Luminescence was measured with a BioTek Synergy 2 Luminometer (BioTek) and normalized to the DMSO-only control. IC ${ }_{50}$ S were calculated using the AAT Bioquest IC50 calculator (https://www.aatbio.com/tools/ic50-calculator).

\section{TUNEL assay}

Apoptotic cells were detected using the terminal deoxynucleotidyl transferase-mediated dUTP nick end labeling (TUNEL assay; Roche Applied Sciences) according to the manufacturer's recommendations.

\section{Reverse transcriptase (RT)-PCR}

RNA extraction (Qiagen RNeasy Mini kit) and RT-PCR was performed as described previously ${ }^{18}$. Exon-overlapping, mRNA/cDNA-specific primers were used to amplify KIT (forward: 5'-TCATGGTCGGATCACAAAGA-3', reverse: 5'-AGGGGCTGCTTCCTAAAGAG-3'; Operon) or $\beta$-actin (forward: 5'-CCAAGGCCAACCGCGAGAAGATGAC-3', reverse: $\quad$ 5'AGGGTACATGGTGGTGCCGCCAGAC-3'), with $\beta$-actin serving as reference gene and loading control.

\section{GIST xenograft model}

GIST-T1 cells were bilaterally injected in the flank of 12 female adult athymic nude mice (BALB/c nude mice; Charles River Laboratories ${ }^{35}$. When tumors were approximately $60 \mathrm{~mm}^{3}$ in size, mice were randomized into groups of 4 animals each for each treatment regimen. In a combined dose-finding/efficacy experiment, homoharringtonine ( $2 \mathrm{mg} / \mathrm{kg}$ or $4 \mathrm{mg} / \mathrm{kg}$ in DMSO) or placebo (solvent control) was administered intraperitoneally (i.p.) twice per week for three weeks. Initial doses were chosen as reported in Kantarjian et al. ${ }^{25}$ and Alvandi et al. ${ }^{28}$. However, $4 \mathrm{mg} / \mathrm{kg}$ was toxic to all animals after one dose and mice treated at $2 \mathrm{mg} / \mathrm{kg}$ also showed signs of toxicity. 
The latter were switched to $1 \mathrm{mg} / \mathrm{kg}$ after the initial treatment, which they tolerated well. The placebo-treated mice showed no signs of toxicity. Tumor volume, weight and general health of the mice were recorded. After the mice were sacrificed, tumors were excised, formalin-fixed and paraffin-embedded (FFPE) for histopathological examination and IHC. Mice were sacrificed using $\mathrm{CO}_{2}$ and cervical dislocation. The animal experiment was approved by the IACUC of the University of Pittsburgh. All experiments were performed in accordance with the relevant guidelines and regulations and in with ARRIVE guidelines.

\section{Statistical analysis}

Statistical significance was assessed using the 2-tailed Student's t-test for independent samples. $P$ values $\leq 0.05$ were considered significant.

\section{RESULTS}

\section{Homoharringtonine inhibits nascent protein synthesis and abrogates KIT protein expression in GIST cells.}

Homoharringtonine $(\mathrm{HHT})$ is a first-in-class mRNA translation elongation inhibitor that is FDA-approved for the treatment of TKI-resistant CML ${ }^{22-25,28}$. To determine whether HHT inhibits protein biosynthesis in GIST cells, nascent protein synthesis was measured by using a fluorescence-based assay that measures incorporation of the methionine analogue Lhomopropargylglycine (HPG) via Click-iT technology and detection with azide-modified Alexa 488 (Fig. 1A). Imatinib (IM)-sensitive (GIST882, GIST-T1) and IM-resistant (GIST48, GIST430) human GIST cell lines was treated with HHT in comparison to the mRNA translation inhibitor cycloheximide and either IM (GIST882, GIST-T1) or sunitinib (SU; GIST430, GIST48), current standard medications for IM-naïve and IM-resistant GIST, respectively. HHT treatment at $1 \mathrm{~h}$ and 
$8 \mathrm{~h}$ led to a substantial decrease in protein translation activity that was comparable to

cycloheximide in all cell lines. While IM and SU also resulted in a reduction of protein synthesis, changes were not as pronounced and more noticeable at the $8 \mathrm{~h}$ time point and therefore likely a secondary effect due to inhibition of the mTOR signaling axis downstream of KIT ${ }^{15}$.

A
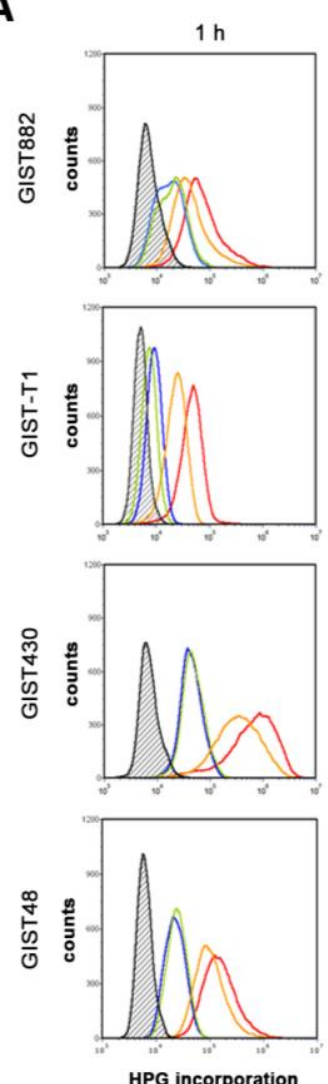

HPG incorporation
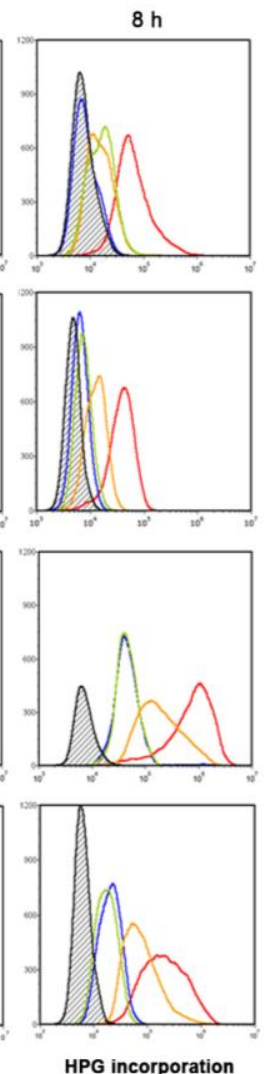
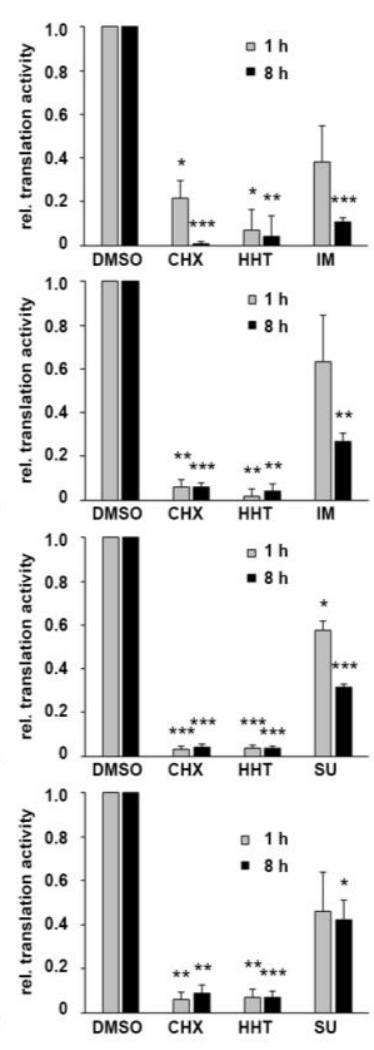

B

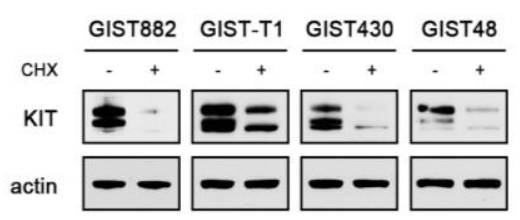

IM-sensitive

IM-resistant
C
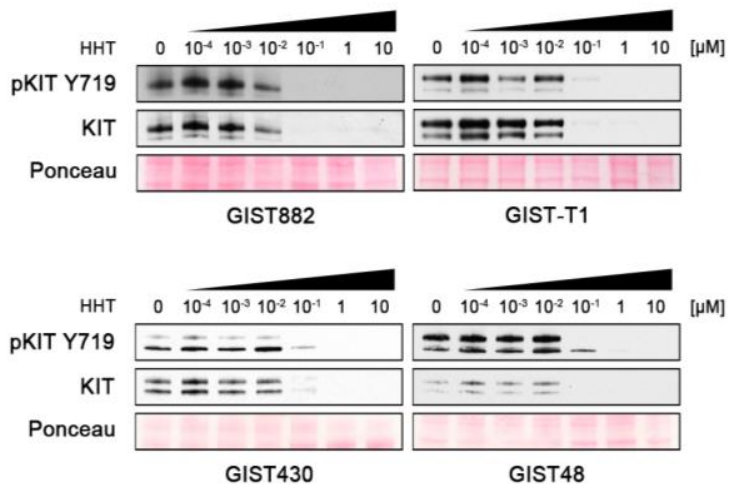

Figure 1. Homoharringtonine inhibits nascent protein synthesis and reduces KIT protein expression in GIST cells. (A) Nascent protein synthesis of GIST cells treated with homoharringtonine (HHT, $0.1 \mu \mathrm{M}$; green), cycloheximide (CHX, $100 \mu \mathrm{g} / \mathrm{ml}$; blue), KIT inhibitor (IM, $1 \mu \mathrm{M}$ for GIST882 and GIST-T1; sunitinib, SU, $1 \mu \mathrm{M}$ for GIST430 and GIST48; orange) or $0.1 \%$ DMSO control (red) for $1 \mathrm{~h}$ or $8 \mathrm{~h}$. Cells were labelled with HPG during the last $30 \mathrm{~min}$ of drug treatment and incorporated cellular HPG linked to azide-modified Alexa488 was quantitated by flow cytometry. Unstained control cells are shown in black in the histogram. A change in nascent protein synthesis is indicated as a relative mean fluorescence value to the DMSO control in the bar graphs. Columns, mean + SE; *, $p<0.05$ in comparison to DMSO control; ${ }^{* *}, p<0.01$ in comparison to control; ${ }^{* *}, p<0.001$ in comparison to control (Student's t-test, 2-tailed). (B) Immunoblot analysis for KIT protein expression of imatinib (IM)-sensitive (GIST882, GIST-T1) and IM-resistant (GIST430, GIST48) GIST cells after treatment with the protein translation inhibitor cycloheximide (CHX; $30 \mu \mathrm{g} / \mathrm{ml}$ for $3 \mathrm{~h}$ ). (C) Immunoblotting for KIT protein expression in IM-sensitive (GIST882, GIST-T1) and IM-resistant (GIST430, GIST48) GIST cells after treatment with HHT for $72 \mathrm{~h}$ at the indicated concentrations. Abbreviations: 882 (GIST882), T1 (GIST-T1), 430 (GIST430), 48 (GIST48). (B, C) Grouped immunoblot images are either cropped from different parts of the same gel or from a separate gel run with another aliquot of the same protein lysate

Many proteins derived from oncogenic driver genes have a high turnover rate and could hence be suitable targets for mRNA translation inhibitors such as $\mathrm{HHT}^{22}$. To determine whether 
this is the case for the mutant KIT protein in GIST cells, levels of KIT expression were determined after inhibition of protein translation with cycloheximide. KIT expression decreased to almost undetectable levels in most cell lines after 3 hours, indicating that the protein has a half-life of less than 1.5 hours in those cells (Fig. 1B). We then tested whether HHT has a similar effect and could show that treatment leads to a dose-dependent reduction of KIT protein expression in IM-sensitive (GIST882, GIST-T1) and IM-resistant (GIST430, GIST48) GIST cells when analyzed by immunoblotting (Fig. 1C).

\section{HHT induces apoptosis and cell cycle arrest in GIST cells}

GIST cell survival is dependent on oncogenic KIT expression and activation ${ }^{1,4,15-19}$. To test whether the substantial downregulation of KIT protein expression after HHT treatment leads to a cytotoxic response, GIST cells were incubated with increasing concentrations of HHT over a 100,000 -fold concentration range $(0.0001 \mu \mathrm{M}$ to $10 \mu \mathrm{M})$. Cell viability and apoptosis were measured using luminescence-based assays and showed robust changes in all cell lines tested (Fig. 2A). IC $\mathrm{C}_{50}$ S ranged from as low as $5 \mathrm{nM}$ (GIST-T1) to $132 \mathrm{nM}$ (GIST48; Table 1).

\begin{tabular}{l|c|} 
& HHT IC \\
\hline Go & $(\mathrm{nM})$ \\
\hline GIST882 & 32 \\
\hline GIST-T1 & 5 \\
\hline GIST430 & 107 \\
\hline GIST48 & 132 \\
\hline
\end{tabular}

Table 1. $\mathrm{IC}_{50}$ (cell viability) values of homoharringtonine (HHT) in imatinib-sensitive and imatinib-resistant GIST cells. Imatinib-sensitive: GIST882, GIST-T1; imatinib-resistant: GIST430, GIST48.

In a dose-response assay, HHT led to the induction of apoptosis (as shown by PARP and caspase 3 cleavage) and cell cycle exit (as shown by a reduction of cyclin A expression) at concentrations as low as $0.1 \mu \mathrm{M}$ (Fig. 2B). The pro-apoptotic effect of HHT was further verified 
by terminal deoxynucleotidyl transferase-mediated dUTP nick end labeling (TUNEL) assay (Fig.

$$
\text { 2C, D). }
$$

A
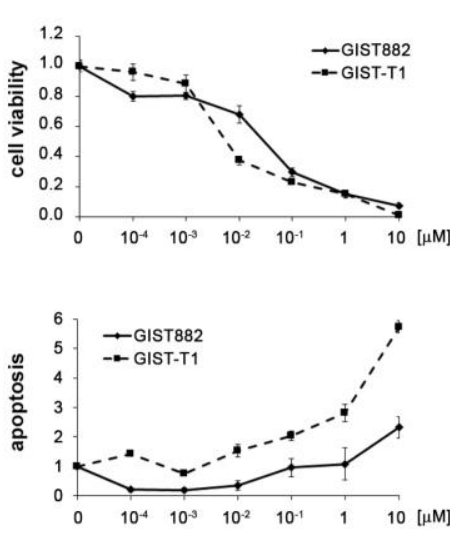

C

C DMSO
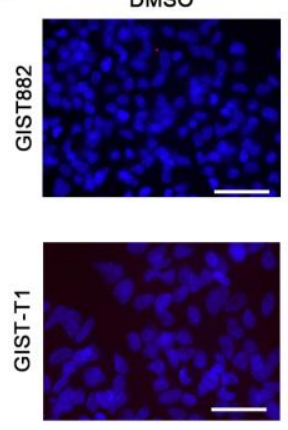

HHT $[0.1 \mu \mathrm{M}]$
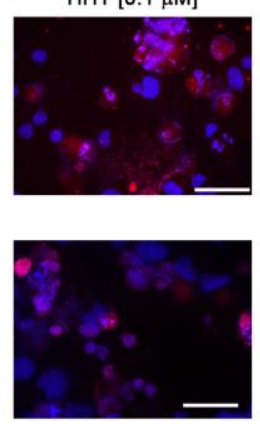
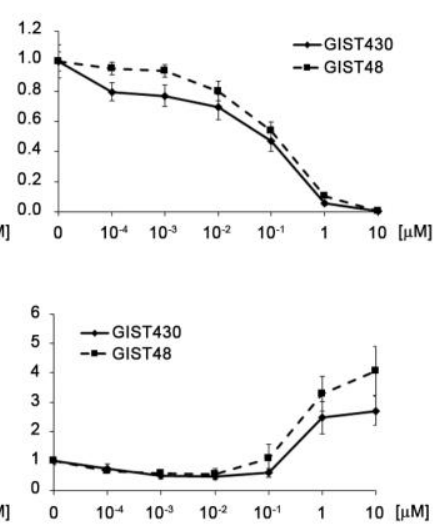

$0[\mu \mathrm{M}]$

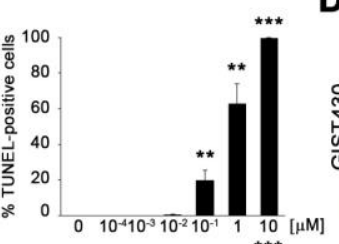

B

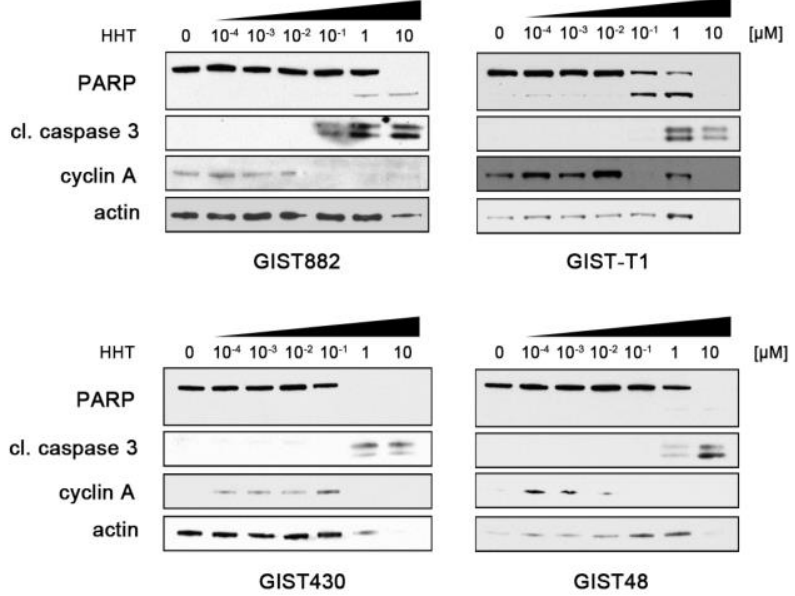

D

DMSO

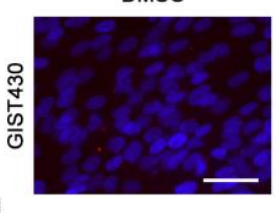

HHT $[0.1 \mu \mathrm{M}]$
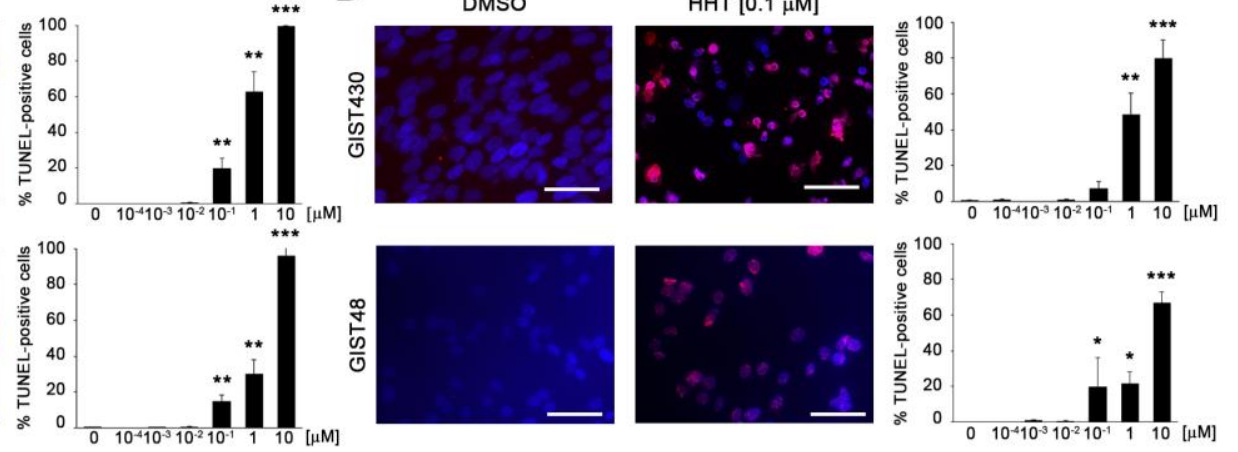

Figure 2. HHT has a pro-apoptotic effect in GIST cells and reduces cell viability in a dose-dependent manner. (A) Dosedependent effect of HHT on cell viability (upper panels) and induction of apoptosis (lower panels) of IM-sensitive (GIST882, GIST-T1; left panels) and IM-resistant (GIST48, GIST430; right panels) GIST cells as measured by luminescence-based assays (mean +/-SE). (B) Immunoblot analysis for markers of apoptosis and cell cycle regulation after treatment of GIST cells with increasing concentrations of HHT $(72 \mathrm{~h})$ as indicated. Grouped immunoblot images are either cropped from different parts of the same gel or from a separate gel run with another aliquot of the same protein lysate. (C, D) Dose-dependent effect of HHT on induction of apoptosis in IM-sensitive (C) and IM-resistant (D) GIST cells as measured by TUNEL assay. Cells were treated for $72 \mathrm{~h}$. Graphs represent mean and standard error of at least two experiments with an average of 500 cells counted per condition. Scale bar, $50 \mu \mathrm{m}$. Columns, mean + SE; *, $\mathrm{p}<0.05$ in comparison to DMSO control; ${ }^{* *}, \mathrm{p}<0.01$ in comparison to control; ${ }^{* * *}$, $\mathrm{p}<0.001$ in comparison to control (Student's t-test, 2-tailed).

\section{The effect of HHT is time-dependent and leads to inhibition of translation initiation}

Having shown that $\mathrm{HHT}$ induces apoptosis and cell cycle exit at a concentration of $0.1 \mu \mathrm{M}$,

we used this concentration to determine the dynamic between translation inhibition and cellular 
response. Levels of total and phosphorylated (Y719) KIT rapidly decreased starting after 1 hour of treatment and were completely lost in all cell lines after 8 to 24 hours (Fig. 3A). Induction of apoptosis as indicated by PARP and caspase 3 cleavage as well as cell cycle exit (reduction of cyclin A levels) was delayed, starting at 24 hours after treatment in most cell lines (Fig. 3B).

A

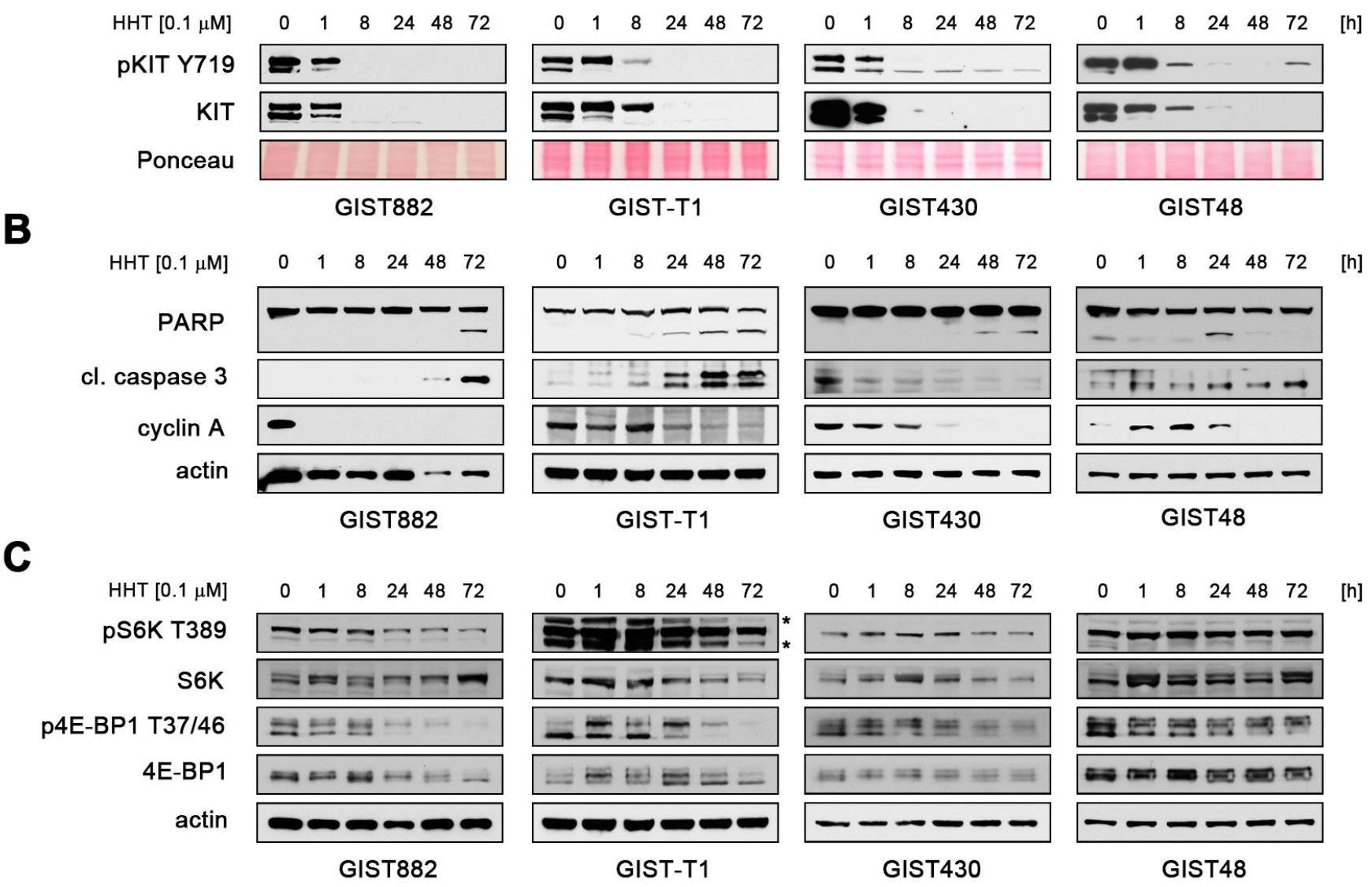

Figure 3. The effect of HHT is time-dependent and leads to secondary inhibition of translation initiation. (A-C) Immunoblot analysis of (A) KIT activation (phosphorylation at Y719) and protein expression, (B) markers of apoptosis and cell cycle regulation and (C) activation of the protein translation regulators ribosomal S6 kinase (S6K; T89) and 4E-BP1 (T37/46) in GIST cells after treatment with DMSO or HHT $(0.1 \mu \mathrm{M})$ for the indicated times. * indicates unspecific band. Grouped immunoblot images are either cropped from different parts of the same gel or from a separate gel run with another aliquot of the same protein lysate.

HHT-treated cells also showed decreased expression and phosphorylation of ribosomal S6 kinase and 4E-BP1, a major regulator of translation initiation factor elF4E (Fig. 3C). Both are 
direct targets of the mTOR kinase, which is downstream of KIT ${ }^{15}$. Taking into account that inhibition of KIT signaling leads to downregulation of nascent protein synthesis (Fig. 1A) and the fact that HHT interferes with the elongation step of protein translation, the above findings likely represent a secondary effect after loss of KIT expression/activation and are not a direct effect of HHT on the translational machinery.

\section{Loss of KIT expression after HHT treatment is not due to reduced mRNA}

\section{transcription}

To further prove that reduced KIT protein expression in GIST cells after HHT treatment is due to the inhibition of protein translation, we analyzed the effect of the drug on mRNA transcription. KIT mRNA levels did not or only minimally decrease with increasing duration of treatment (Fig. 4), thus further corroborating the notion that HHT's main mechanism of action is the inhibition of protein translation.

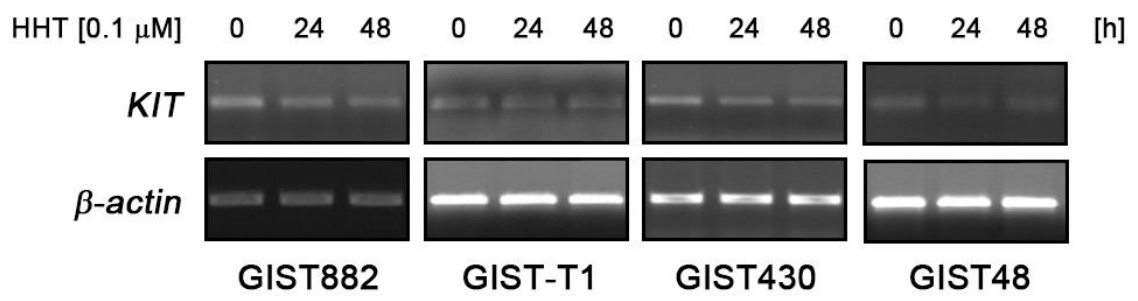

Figure 4. KIT mRNA levels are not affected by HHT treatment. RT-PCR of KIT mRNA expression after treatment of GIST cells with DMSO or $0.1 \mu \mathrm{M} \mathrm{HHT}$ for the indicated times. $\beta$-actin mRNA expression serves as loading control.

\section{HHT has in vivo antitumor activity in a GIST xenograft}

To determine the antitumoral activity of HHT in vivo, we used a GIST xenograft model (GIST-T1). Combined dose finding and efficacy testing showed toxicity at higher doses. However, animals treated with low-dose HHT $(1 \mathrm{mg} / \mathrm{kg})$ showed a remarkable tumor shrinkage. Over the course of three weeks, HHT treatment resulted in a marked histopathologic response with large 
A
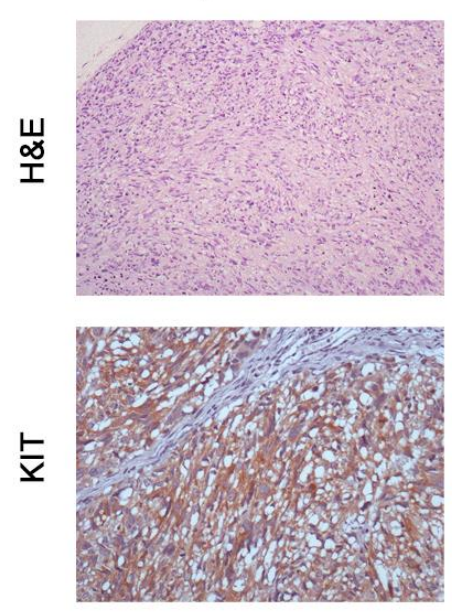

C

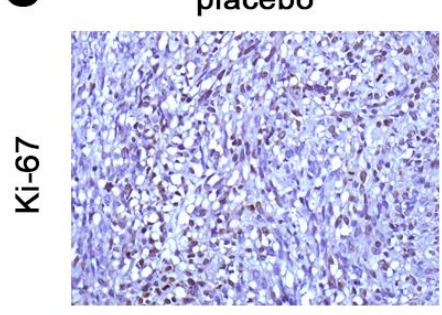

D

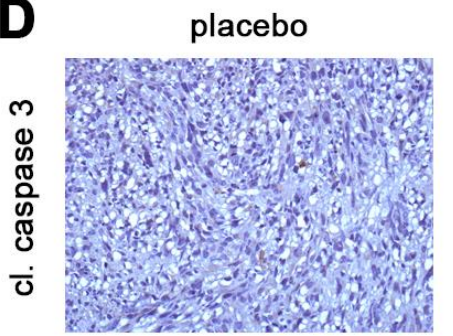

$\mathrm{HHT}$
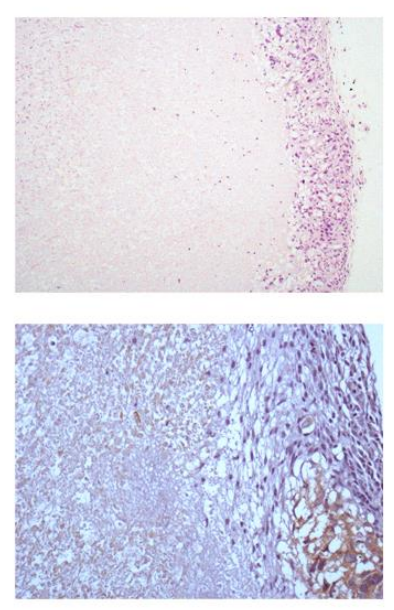

HHT

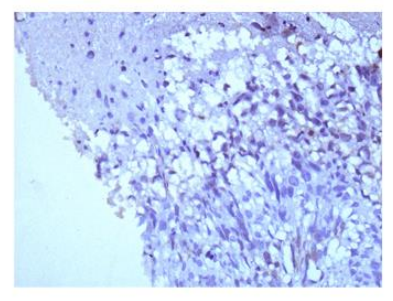

HHT

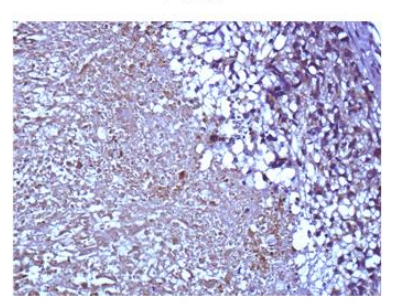

B
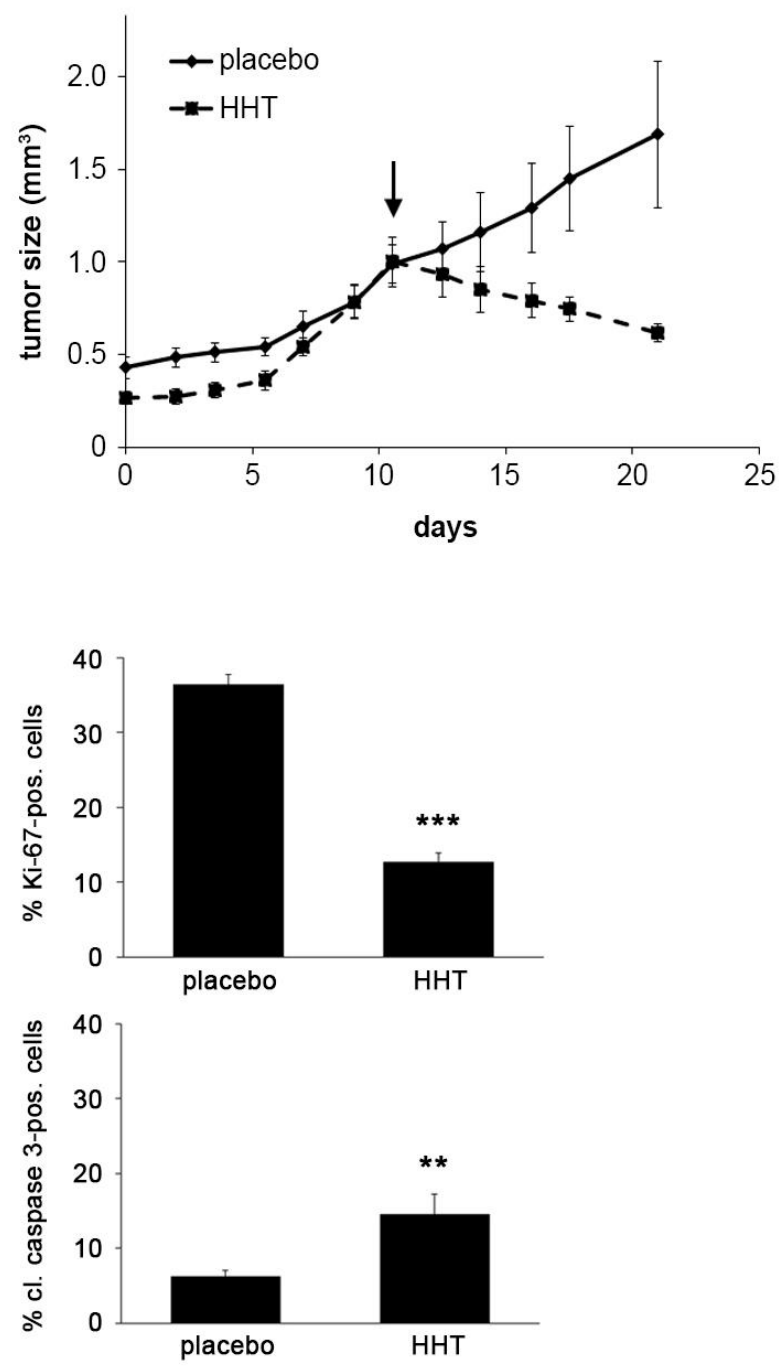

Figure 5. HHT is effective in a GIST xenograft. (A) Histopathologic response (hematoxylin and eosin, H\&E; 10X; upper panels) and immunohistochemical staining for KIT (20X; lower panels) of GIST-T1 xenografts after treatment with HHT in comparison to placebo-treated controls. (B) Relative tumor volume of GIST-T1 xenografts since inoculation and over the course of a 21-day treatment with vehicle (solid line) or HHT (dashed line). Measurements represent the average of four tumors per group treated either with placebo or $2 \mathrm{mg} / \mathrm{kg} \mathrm{HHT}$ (reduced to $1 \mathrm{mg} / \mathrm{kg}$ at day 7) for 21 days. Arrow indicates start of treatment. (C,D) Immunohistochemical staining for (C) Ki-67 (20X; upper panels) and (D) cleaved caspase 3 (20X; lower panels) of GIST-T1 xenografts treated with HHT or placebo control. Graphs represent mean and standard error of positive cells in 10 high power fields (HPF; 400-fold magnification), with an average of 245 cells counted per HPF. Columns, mean + SE; ${ }^{* *}, p \leq 0.01$ in comparison to control; ${ }^{* * *}, p \leq 0.001$ in comparison to placebo control (Student's t-test, 2-tailed).

areas of central necrosis (Fig. 5A, upper panels). As expected from our in vitro studies, HHTtreated tumor cells showed a loss of KIT expression (Fig. 5A, lower panels). This was accompanied by significant tumor reduction, while placebo-treated xenografts continued to grow 
exponentially (Fig. 5B). Furthermore, HHT led to a significant reduction of proliferation (Fig. 5C; reduction of the Ki-67-positive proliferation fraction from $36.4 \%$ in placebo-treated controls to 12.8\% in HHT-treated tumors) and a significantly increased proportion of apoptotic cells (cleaved caspase 3-positive) from $6.3 \%$ in vehicle-treated controls to $14.6 \%$ in HHT-treated animals (Fig. 5D). Taken together, HHT has significant activity in GIST xenografts.

In summary, we show that HHT is effective in IM-sensitive and IM-resistant GIST cells as well as a GIST xenograft model. Its mechanism of action involves a substantial downregulation of KIT expression and subsequent inhibition of downstream signaling pathways and induction of apoptosis through inhibition of nascent protein biosynthesis.

\section{DISCUSSION}

GISTs can be successfully treated with tyrosine kinase inhibitors (TKIs) that target KIT, such as imatinib mesylate, but many patients develop resistance to imatinib as well as to secondor later-line TKIs ${ }^{5,6}$. Mechanistically, TKI resistance in GIST is largely mediated through secondary KIT mutations, indicating that active KIT continues to be the main driver of the disease during tumor progression 7,8 . Because of the intratumoral heterogeneity of those mutations, treatment responses may be uneven and lead to incomplete responses ${ }^{20}$. Targeting KIT by inhibiting protein translation is independent of the KIT mutational status and may hence lead to more complete and durable therapeutic effects.

In this study, we show that homoharringtonine $(\mathrm{HHT})$, a first-in-class inhibitor of protein biosynthesis at the step of peptide chain elongation, has significant antitumoral activity in GIST cells. This was independent of the KIT mutational status and imatinib sensitivity. Evaluating several human GIST cell line models, HHT potently inhibited nascent protein synthesis, KIT protein expression and phosphorylation as well as downstream signaling. Apoptosis was induced 
in a dose- and time-dependent manner via induction of PARP and caspase 3/7 cleavage. At the same time, the decreased levels of cyclin A point to HHT-induced cell cycle arrest in S phase. As expected, expression of KIT mRNA expression was largely unaffected. Results from a GIST xenograft model show antitumor activity of HHT in vivo. Taken together, our results indicate that HHT has potent antitumor activity in GIST in vitro and in vivo, and encourage clinical studies in GIST patients.

The fact that HHT has activity in imatinib-resistant cells makes it a promising medication for use in the TKI-resistant setting. Although two new compounds have been recently approved (ripretinib [Qinlock®; Deciphera] and avapritinib [Ayvakit®; Blueprint Medicines]), resistance to both drugs still occurs ${ }^{12-14,36}$, and further therapeutic options are needed. In addition, avapritinib's indication only covers PDGFRA D842V-mutated GIST, limiting its use to a very small patient population. Based on its mechanism of action, it is conceivable that HHT is also effective in PDGFRA-mutant GIST ${ }^{3}$, including mutations other than $\mathrm{D} 842 \mathrm{~V}$, for which only few therapeutic options are available.

Mechanistically, it is highly likely that the loss of KIT expression induced by HHT is the main mediator of GIST cell death. GIST cells are dependent on active KIT signaling for their survival, and we have previously shown that knocking down KIT expression with small interfering RNA (siRNA) leads to a pronounced apoptotic response via a caspase-3-dependent mechanism 16,37. Similarly, Jin et al. reported significantly increased apoptosis after silencing KIT in two KITmutant mastocytosis models ${ }^{31}$. Likewise, these cells were highly sensitive to treatment with HHT. In our study, loss of KIT expression induced by HHT also led to secondary changes that occurred delayed when compared to loss of KIT expression, but likely contributed to drug efficacy. For example, decreased phosphorylation of ribosomal S6 kinase and the translation initiation factor 4E-BP1, both downstream of the $\mathrm{KIT} / \mathrm{mTOR}$ signaling axis, point to an inhibition of translation initiation in addition to HHT-mediated inhibition of the elongation step of protein translation ${ }^{15}$. Other factors of HHT-mediated apoptosis, such as signal transducer and activator of transcription 
(STAT) proteins, have been reported ${ }^{38,39}$. However, the respective STAT proteins are either not expressed or not regulated in a KIT-dependent fashion in the models used in our current study ${ }^{15}$.

Based on our results, it would not be expected that "KIT-negative" GISTs, such as those KIT-mutant tumors that lose KIT expression during the course of TKI treatment are sensitive to $\mathrm{HHT}^{40,41}$. However, it is conceivable that a presumed alternate oncogenic driver has a high turnover rate making it amenable to inhibition of protein biosynthesis ${ }^{42}$. Preliminary results from our laboratory (data not shown) suggest that this might be the case.

Clinical trials to evaluate HHT's efficacy in GIST patients are warranted and feasible. The drug is FDA-approved for TKI-resistant $\mathrm{CML}$, and $\mathrm{IC}_{50}$ S values of our preclinical GIST models (55 - $132 \mathrm{nM}$ ) are comparable to those reported of CML in vitro models (20 - $150 \mathrm{ng} / \mathrm{ml}$, equivalent to $67-500 \mathrm{nM})^{43,44}$. Current HHT dosing schedules include subcutaneous self-application as opposed to intravenous infusion ${ }^{26}$, which might be of importance to a patient population that is accustomed to oral anti-cancer medications. It is still possible, however, that preclinical results do not translate into the clinic. Solid tumors, such as GIST, sometimes respond differently to the same drug than hematological malignancies, as has been reported for the proteasome inhibitor bortezomib (Velcade $\left.{ }^{\circledR}\right){ }^{45,46}$. The fact that $\mathrm{HHT}$ is a global inhibitor of protein biosynthesis might translate into adverse effects, such as those reported in CML patients, like pancytopenia and diarrhea $^{47}$.

Taken together, we have shown that the protein translation inhibitor HHT is highly effective in GIST cells in vitro and in vivo and leads to a loss of KIT expression. Further studies to bring the drug into the clinic are warranted.

\section{ACKNOWLEDGEMENTS}

The authors would like to thank Jonathan A. Fletcher for sharing important reagents, Stefan Duensing for critically reading the manuscript and members of the Pittsburgh Sarcoma 
Stefan Duensing for critically reading the manuscript and members of the Pittsburgh Sarcoma.

\section{GRANT SUPPORT:}

This work was supported by the GIST Cancer Research Fund (to A.D.), The Life Raft Group (to A.D.), the Out of the Woods Foundation (A.D.), Pittsburgh Cure Sarcoma (A.D.), NIH P50CA121973 (University of Pittsburgh Skin Cancer Specialized Program of Research Excellence; M.S.), the Hillman Foundation (M.S.), a Klionsky Fellowship (Department of Pathology, University of Pittsburgh School of Medicine; to C.L.) and private donations (A.D.). This project used the UPMC Hillman Cancer Center and Tissue and Research Pathology/Pitt Biospecimen Core shared resource which is supported in part by award P30CA047904. A.D. and M.S. are supported by UPMC Hillman Cancer Center and in part by a grant from the Pennsylvania Department of Health. The Department specifically disclaims responsibility for any analyses, interpretations or conclusions.

\section{DISCLOSURE OF POTENTIAL CONFLICTS OF INTEREST}

None.

\section{AUTHOR CONTRIBUTIONS}

\section{Conception and design: A. Duensing}

Development of methodology: D.M. Lee, S. Patil, A. Rao, L. Liu, P. Trent, A.A. Ali, C. Liu, J.L. Rausch, M. Shuda, A. Duensing

Acquisition of data: D.M. Lee, A. Sun, S. Patil, A. Rao, L. Liu, P. Trent, A.A. Ali, C. Liu, J.L. Rausch, N. Amankulor, M. Shuda, A. Duensing 
Analysis and interpretation of data: D.M. Lee, A. Sun, S. Patil, A. Rao, P. Trent, A.A. Ali, J.L. Rausch, C. Liu, M. Shuda, N. Amankulor, A. Duensing

Writing, review and/or revision of the manuscript: D.M. Lee, A. Sun, S. Patil, L. Liu, A. Rao, C. Liu, P. Trent, A.A. Ali, C. Liu, J.L. Rausch, L. Presutti, N. Amankulor, M. Shuda, A. Duensing

Administrative, technical, or material support: A. Sun, L., Presutti, A. Duensing

Study supervision: A. Duensing 


\section{REFERENCES}

1. Hirota, S. et al. Gain-of-function mutations of c-kit in human gastrointestinal stromal tumors. Science 279, 577-580 (1998).

2. Rubin, B. P. et al. KIT activation is a ubiquitous feature of gastrointestinal stromal tumors. Cancer Res. 61, 8118-8121 (2001).

3. Heinrich, M. C. et al. PDGFRA activating mutations in gastrointestinal stromal tumors. Science 299, 708-710 (2003).

4. Tuveson, D. A. et al. STI571 inactivation of the gastrointestinal stromal tumor c-KIT oncoprotein: biological and clinical implications. Oncogene 20, 5054-5058 (2001).

5. Demetri, G. D. et al. Efficacy and safety of imatinib mesylate in advanced gastrointestinal stromal tumors. N. Engl. J. Med. 347, 472-480 (2002).

6. Verweij, J. et al. Progression-free survival in gastrointestinal stromal tumours with highdose imatinib: randomised trial. Lancet 364, 1127-1134 (2004).

7. Antonescu, C. R. et al. Acquired resistance to imatinib in gastrointestinal stromal tumor occurs through secondary gene mutation. Clin. Cancer Res. 11, 4182-4190 (2005).

8. Wardelmann, E. et al. Polyclonal evolution of multiple secondary KIT mutations in gastrointestinal stromal tumors under treatment with imatinib mesylate. Clin Cancer Res 12, 1743-1749 (2006).

9. Demetri, G. D. et al. Efficacy and safety of sunitinib in patients with advanced gastrointestinal stromal tumour after failure of imatinib: a randomised controlled trial. Lancet 368, 1329-1338 (2006).

10. George, S. et al. Efficacy and safety of regorafenib in patients with metastatic and/or unresectable GI stromal tumor after failure of imatinib and sunitinib: a multicenter phase II trial. J. Clin. Oncol. 30, 2401-2407 (2012).

11. Demetri, G. D. et al. Efficacy and safety of regorafenib for advanced gastrointestinal 
stromal tumours after failure of imatinib and sunitinib (GRID): an international, multicentre, randomised, placebo-controlled, phase 3 trial. Lancet 381, 295-302 (2013).

12. Blay, J. Y. et al. Ripretinib in patients with advanced gastrointestinal stromal tumours (INVICTUS): a double-blind, randomised, placebo-controlled, phase 3 trial. Lancet Oncol. 21, 923-934 (2020).

13. Dhillon, S. Avapritinib: First Approval. Drugs 80, 433-439 (2020).

14. Jones, R. L. et al. Avapritinib in unresectable or metastatic PDGFRA D842V-mutant gastrointestinal stromal tumours: Long-term efficacy and safety data from the NAVIGATOR phase I trial. Eur. J. Cancer 145, 132-142 (2021).

15. Duensing, A. et al. Mechanisms of oncogenic KIT signal transduction in primary gastrointestinal stromal tumors (GISTs). Oncogene 23, 3999-4006 (2004).

16. Rausch, J. L. et al. Opposing roles of KIT and ABL1 in the therapeutic response of gastrointestinal stromal tumor (GIST) cells to imatinib mesylate. Oncotarget 8, 4471-4483 (2017).

17. Bauer, S., Yu, L. K., Demetri, G. D. \& Fletcher, J. A. Heat shock protein 90 inhibition in imatinib-resistant gastrointestinal stromal tumor. Cancer Res. 66, 9153-9161 (2006).

18. Boichuk, S. et al. Unbiased compound screening identifies unexpected drug sensitivities and novel treatment options for gastrointestinal stromal tumors. Cancer Res. 74, 1200$1213(2014)$.

19. Bauer, S. et al. Proapoptotic activity of bortezomib in gastrointestinal stromal tumor cells. Cancer Res. 70, 150-159 (2010).

20. Serrano, C. et al. Complementary activity of tyrosine kinase inhibitors against secondary kit mutations in imatinib-resistant gastrointestinal stromal tumours. Br. J. Cancer 120, (2019).

21. Rausch, J. L. et al. Differential antitumor activity of compounds targeting the ubiquitinproteasome machinery in gastrointestinal stromal tumor (GIST) cells. Sci. Rep. 10, 1-14 
(2020).

22. Gandhi, V., Plunkett, W. \& Cortes, J. E. Omacetaxine: A Protein Translation Inhibitor for Treatment of Chronic Myelogenous Leukemia. Clin. Cancer Res. 20, 1735-1740 (2014).

23. Huang, M. T. Harringtonine, an inhibitor of initiation of protein biosynthesis. Mol Pharmacol 11, 511-519 (1975).

24. Tujebajeva, R. M., Graifer, D. M., Karpova, G. G. \& Ajtkhozhina, N. A. Alkaloid homoharringtonine inhibits polypeptide chain elongation on human ribosomes on the step of peptide bond formation. FEBS Lett. 257, 254-256 (1989).

25. Kantarjian, H. M. et al. Homoharringtonine - History, current research, and future directions. Cancer 92, 1591-1605 (2001).

26. Winer, E. S. \& DeAngelo, D. J. A Review of Omacetaxine: A Chronic Myeloid Leukemia Treatment Resurrected. Oncol. Ther. 6, 9-20 (2018).

27. FRESNO, M., JIMÉNEZ, A. \& VÁZQUEZ, D. Inhibition of Translation in Eukaryotic Systems by Harringtonine. Eur. J. Biochem. 72, 323-330 (1977).

28. Alvandi, F. et al. U.S. Food and Drug Administration Approval Summary: Omacetaxine Mepesuccinate as Treatment for Chronic Myeloid Leukemia. Oncologist 19, 94-99 (2014).

29. Wolff, N. C. et al. High-throughput simultaneous screen and counterscreen identifies homoharringtonine as synthetic lethal with von Hippel-Lindau loss in renal cell carcinoma. Oncotarget 6, 16951-16962 (2015).

30. Howard, T. P. et al. Rhabdoid Tumors Are Sensitive to the Protein-Translation Inhibitor Homoharringtonine. Clin. Cancer Res. 26, 4995-5006 (2020).

31. Jin, Y. et al. The antitumor activity of homoharringtonine against human mast cells harboring the KIT D816V mutation. Mol. Cancer Ther. 9, 211-223 (2010).

32. Duensing, S. et al. Cyclin-dependent kinase inhibitor indirubin-3'-oxime selectively inhibits human papillomavirus type 16 E7-induced numerical centrosome anomalies. Oncogene 23, 8206-8215 (2004). 
33. Taguchi, T. et al. Conventional and molecular cytogenetic characterization of a new human cell line, GIST-T1, established from gastrointestinal stromal tumor. Lab. Invest. 82, 663665 (2002).

34. Shuda, M. et al. CDK1 substitutes for mTOR kinase to activate mitotic cap-dependent protein translation. Proc. Natl. Acad. Sci. 112, 5875-5882 (2015).

35. Zhang, X. et al. IDH mutant gliomas escape natural killer cell immune surveillance by downregulation of NKG2D ligand expression. Neuro. Oncol. 18, 1402-1412 (2016).

36. Grunewald, S. et al. Resistance to avapritinib in PDGFRA-driven GIST is caused by secondary mutations in the PDGFRA kinase domain. Cancer Discov. 11, CD-20-0487 (2020).

37. Liu, Y. et al. Histone H2AX is a mediator of gastrointestinal stromal tumor cell apoptosis following treatment with imatinib mesylate. Cancer Res. 67, 2685-2692 (2007).

38. Cao, W. et al. Homoharringtonine induces apoptosis and inhibits STAT3 via IL6/JAK1/STAT3 signal pathway in Gefitinib-resistant lung cancer cells. Sci. Rep. 5, 8477 (2015).

39. Lindqvist, L. M. et al. Translation inhibitors induce cell death by multiple mechanisms and Mcl-1 reduction is only a minor contributor. Cell Death Dis. 3, e409-9 (2012).

40. Bauer, S. et al. Resection of residual disease in patients with metastatic gastrointestinal stromal tumors responding to treatment with imatinib. Int J Cancer 117, 316-325 (2005).

41. Debiec-Rychter, M. et al. Mechanisms of resistance to imatinib mesylate in gastrointestinal stromal tumors and activity of the PKC412 inhibitor against imatinib-resistant mutants. Gastroenterology 128, 270-279 (2005).

42. Tu, Y. et al. Activated tyrosine kinases in gastrointestinal stromal tumor with loss of KIT oncoprotein expression. Cell Cycle 17, 2577-2592 (2018).

43. Visani, G. et al. Effects of homoharringtonine alone and in combination with alpha interferon and cytosine arabinoside on 'in vitro' growth and induction of apoptosis in chronic myeloid 
leukemia and normal hematopoietic progenitors. Leukemia 11, 624-628 (1997).

44. Quintás-Cardama, A., Kantarjian, H. \& Cortes, J. Homoharringtonine, Omacetaxine Mepesuccinate, and Chronic Myeloid Leukemia Circa 2009. doi:10.1002/cncr.24601

45. Maki, R. G. et al. A multicenter Phase II study of bortezomib in recurrent or metastatic sarcomas. Cancer 103, 1431-1438 (2005).

46. Caravita, T., de Fabritiis, P., Palumbo, A., Amadori, S. \& Boccadoro, M. Bortezomib: Efficacy comparisons in solid tumors and hematologic malignancies. Nature Clinical Practice Oncology 3, 374-387 (2006).

47. Safety Profile and Adverse Reactions | SYNRIBO (omacetaxine mepesuccinate). Available at: https://www.synribohcp.com/Adverse_Reactions. (Accessed: 5th August 2021) 